\title{
Editorial: An Introduction to the Special Section on International Perspectives on Cultural and Social Issues
}

\author{
Christoph Bördlein ${ }^{1} \cdot$ Sarah M. Richling ${ }^{2} \cdot$ Holly Seniuk $^{3}$
}

Published online: 2 November 2021

(c) Association for Behavior Analysis International 2021

In its over 40-year history, Behavior and Social Issues has published articles on a wide range of topics related to important social issues such as environmental sustainability, criminal justice, collective violence, and diversity and bias. These are topics that are rarely or have only been recently covered in other behavior-analytic journals. In 2019, Behavior and Social Issues became an official publication of the Association for Behavior Analysis International (ABAI) and continues to publish peer-reviewed, scholarly articles using cultural analysis and behavior science to address large-scale social issues occurring across the globe (Luke et al., 2017).

In this special section, the international focus of the organization and this journal is highlighted by behavior analysts, as well as scholars and researchers from related disciplines, contributing scholarly work occurring outside of the United States. Submissions were received from across the globe, from both countries commonly associated with the adoption of behavior analysis and countries where the community of behavior analysts is small. This special issue includes submissions addressing behavior-analytic topics in Brazil, the Czech Republic, Europe (broadly), France, and Austria. Nevertheless, this selection is not representative of behavior analysis worldwide, as important geographical regions such as Japan, China, Mexico, and Colombia are not included here. The articles included are only those submitted for this call. We hope in the future to publish contributions from many of those places not yet represented. Moreover, we hope to see behavior analysis expand more globally into areas such as Africa, which do not currently have established ABAI-affiliated chapters.

The overall purpose of this special section is to provide a platform for behavior analysts, internationally, to share their work addressing behavior-analytic practice and social and cultural research globally. The articles included in this special section

Sarah M. Richling

smr0043@auburn.edu

1 University of Applied Sciences Würzburg-Schweinfurt, Würzburg, Germany

2 Department of Psychological Sciences, Auburn University, 226 Thach Hall, Auburn University, Auburn, AL 36849-5214, USA

3 Behavior Analyst Certification Board, Littleton, CO, USA 
were selected for their scholarly focus on (a) interdisciplinary efforts to produce large-scale change at the systems, community, or cultural levels in the area of education; (b) systemic and conceptual discussions of social issues unique to a specific region conducted by scholars who have intimate knowledge of the relevant contextual factors of that region (e.g., feminism and racism); (c) historical and contemporary accounts of behavioral science in international locations; and (d) discussions of unique barriers for behavior analysts addressing social issues in their respective countries.

Around the world, behavior analysis is predominantly applied to the treatment of autism, which has been a gateway for the discipline in many countries (see DeSouza \& Konrad; Kingsdorf \& Pančocha; and Turgeon \& Lanovaz, this issue). RollPettersson et al. (2020) provided a history of behavior analysis and higher education in Europe, focusing on the United Kingdom, Northern Ireland, the Czech Republic, Greece, Iceland, Italy, Norway, and Sweden. Although the focus of the article is on the history of the application of behavior analysis as a treatment for autism in those countries, it is clear this area of focus has played an important role in the advancement of behavior analysis. In some countries, this has led to the field of behavior analysis often being synonymous with behavior-based autism treatment. For example, the German Wikipedia entry for "applied behavior analysis" is exclusively about the treatment of autism (Applied Behavior Analysis, 2021). As highlighted by Konrad and DeSouza (this issue) and Turgeon and Lanovaz (this issue), the impact of specific contextual and cultural factors related to the effective delivery of behavior-analytic services is an area in need of further discussion and investigation. We applaud our colleagues across the globe who have made tremendous progress in advancing the science of behavior while also supporting individuals with a diagnosis of autism. We also recognize it is important to acknowledge the progress that has been, and is being, made by those colleagues in other areas of social importance (e.g., climate change, racism, and gender issues).

The development of behavior analysis, particularly as it pertains to social issues, outside of the United States involves a lineage of pioneers who paved the way in their respective country or region. Norway and a few additional countries in Europe have an active academic behavior-analytic scene (see Arntzen \& Pellon, this issue). Brazil also has a rich history of applying behavior analysis to cultural issues (see de Almeida; Moreira et al.; and Todorov et al., this issue). For example, in Brazil, the early work of Fred S. Keller inspired several theses and dissertations. Albuquerque et al. (this issue) highlight ever-increasing trends in these academic products in Brazil, indicating a commitment to expanding the field of behavior analysis into the future. Todorov et al. (this issue) discuss the growing interest in the application of behavior analysis to culture and note that it led to the development of three think tanks where researchers from Brazil, the United States, and Norway gathered to discuss issues related to the behavior analysis of culture. The authors provide a timeline of the major events, and the important people, that led to the observed expansion of behavior analysis in Brazil.

Arntzen and Pellon (this issue) provide an analysis of important events such as conferences and the formation of various organizations that have had a significant impact on both the European Association for Behavior Analysis and the European 
Journal of Applied Behavior Analysis. The authors also present a figure to show the location of various educational training programs in behavior analysis across Europe and make the argument that although behavior analysis is spreading across Europe, there is still work to be done in terms of furthering its reach.

Behavior and Social Issues has a long history of publishing articles from researchers outside of North America. However, these articles are published alongside manuscripts from North American authors. Therefore, information about, or attention paid to, the cultural contexts in which the work is being conducted may be overshadowed by the topics themselves. Although the topics of the articles may be of similar importance to North American authors, the differences in the cultural contexts provide unique opportunities to generate new research and application ideas and foster new, international collaborations.

Behavior analysts worldwide are striving to make this world a better place by changing the (physical and social) environment in which behaviors occur. The recent threat of the COVID-19 pandemic to citizens around the world illuminates the direct link between public health and human behavior on a global scale. At this level, there are many ways in which behavior-analytic research and practice can influence public health outcomes (e.g., Bördlein, 2020; Lillie et al., 2021; Normand et al., 2021; Winett et al., 1991). For example, Santos Marques and de Almeida (this issue) analyzed statements related to COVID-19 made by the president of Brazil. The results suggested that there was a high frequency of ineffective statements and statements that contradicted the recommendations of experts. The authors suggest that a behavior-scientific analysis of governmental practices can inform the development of public policies. Many other critical challenges beyond the pandemic are currently faced by citizens around the globe, not just in the United States, including those typically addressed through social work (see Friman, 2021; Gambrill, 1995; Thyer, 1987). As with the pandemic, it will take a global effort to advance the science of behavior as a potential solution to global-scale cultural phenomena. The ability of culturo-behavior science to aid in these endeavors can be increased, we believe, by facilitating collaboration among behavior analysts, irrespective of country, through our common language of science.

Among the collection of articles in this special section, common themes emerge, including racial injustice, gender issues, and education, all of which can resonate with readers regardless of their geographic location. None of these issues are confined within the borders of a single country. Understanding what stands in the way of addressing issues such as gender equality and deriving effective responses should be viewed and analyzed differently among different countries and cultures (see, e.g., Lira et al.; Mizael; and Mizael et al., this issue). Valuable work toward addressing important social issues is taking place around the world. Critical next steps include finding ways to enhance the dissemination of this work and creating systems and environments that foster international collaboration to do so. Mizael (this issue) suggests that language and severe consequences for those who engage in activism are two of the primary barriers that Brazilian researchers face when working to address social issues. It is likely that these barriers are widespread. Actions directly analyzing and addressing these barriers may help create and support the systems that will facilitate progress. The goal is not necessarily to bring behavior analysis to as many 
countries as possible, but to identify how and where the whole of behavior science-its conceptual and theoretical foundations, the experimental science, applied behavior analysis, and the practice of behavior analysis - can aid in improving conditions of life worldwide, particularly with respect to social issues.

Author Note Our names are listed in alphabetical order, as all of us are regarded as having contributed equally to this submission. This is not an official position of the Behavior Analyst Certification Board.

\section{Declarations}

Conflict of Interest We have no known conflicts of interest to disclose.

\section{References}

Applied Behavior Analysis. (2021, November 1). In Wikipedia. https://de.wikipedia.org/wiki/Applied_ Behavior_Analysis

Bördlein, C. (2020). Promoting hand sanitizer use in a university cafeteria. Behavior and Social Issues, 29, 255-263. https://doi.org/10.1007/s42822-020-00026-y

Friman, P. C. (2021). There is no such thing as a bad boy: The circumstances view of problem behavior. Journal of Applied Behavior Analysis. Advance online publication. https://doi.org/10.1002/jaba.816

Gambrill, E. (1995). Behavioral social work. Research on Social Work Practice, 5(4), 460-484. https:// doi.org/10.1177/104973159500500406

Lillie, M. A., Harman, M. J., Hurd, M., \& Smalley, M. R. (2021). Increasing passive compliance to wearing a facemask in children with autism spectrum disorder. Journal of Applied Behavior Analysis, 54(2), 582-599. https://doi.org/10.1002/jaba.829

Luke, M. M., Roose, K. M., Rakos, R. F., \& Mattaini, M. A. (2017). The history and current status of Behavior and Social Issues: 1978-2016. Behavior and Social Issues, 26, 111-127. https://doi.org/ 10.5210/bsi.v.26i0.7728

Normand, M. P., Dallery, J., \& Slanzi, C. M. (2021). Leveraging applied behavior analysis research and practice in the service of public health. Journal of Applied Behavior Analysis, 54(2), 457-483. https://doi.org/10.1002/jaba.832

Roll-Pettersson, L., Gena, A., Eldevik, S., Moderato, P., Sigurdardottir, Z. G., Dillenburger, K., Keenan, M., \& Ala'i-Rosales, S. (2020). Higher education and behavior analysis in Europe: Creating a unified approach for the training of autism professionals. European Journal of Behavior Analysis, 21(1), 158-184. https://doi.org/10.1080/15021149.2020.1758990

Thyer, B. A. (1987). Behavioral social work. The Behavior Therapist, 10(6), 131-134.

Winett, R. A., Moore, J. F., \& Anderson, E. S. (1991). Extending the concept of social validity: Behavior analysis for disease prevention and health promotion. Journal of Applied Behavior Analysis, 24(2), 215-230. https://doi.org/10.1901/jaba.1991.215 